Marketing in Asia Group

\title{
Zhong-Yong Thinking and Employees' Behaviour at the Workplace: Setting a Research Agenda
}

\author{
Binyao Ning \\ Azman Hashim International Business School, Universiti Teknologi Malaysia, \\ Malaysia \\ Shaoguan University, Guangdong, China

\section{Rosmini Omar} \\ Azman Hashim International Business School, Universiti Teknologi Malaysia, \\ Malaysia \\ Ye Ye \\ Azman Hashim International Business School, Universiti Teknologi Malaysia, \\ Malaysia
}

\begin{abstract}
As the essence of traditional Chinese culture, Zhong-Yong is closely related to the psychology, behaviour, and life of Chinese people. Due to the advent of indigenous psychological research, several studies have explored Zhong-Yong thinking (ZYT) in recent decades, but such research is still in its infancy. Although some scholars have affirmed that the influence of ZYT on the Chinese people, the systematic synthesis of the impact of Zhong-Yong thinking on employee's behaviour at the workplace is limited. Based on the established literature, this study offers a systematic review of 22 selected articles in the field by conducting a bibliometric analysis to categorise the three main clusters of innovative behaviour, voice behaviour, and employee performance. The findings of this study enable future researchers to gain a deeper understanding and enrich the field by offering a wide range of perspectives on the direction of research. Theoretical and practical implications for Asian business context are provided.
\end{abstract}

Keywords: Zhong-Yong Thinking, Bibliometric Analysis, Chinese Indigenous Research, Employee Behaviour, Innovation Behaviour, Voice Behaviour 


\section{Introduction}

The collision of Asian and Western cultures under the globalization has led scholars and practitioners in reassessing the adequacy of the mainstream theories of social behaviour based on Western society to explain the phenomenon of employees' behaviour in Asian countries. For example, Academy of Management Journal, Management and Organisation Review and Asia Pacific Journal of Management have discussed special issues on indigenous management research (Chiu, 2017; Li et al., 2016; Li et al., 2019). In recent years, Chinese enterprises have increasingly entered the world in the form of globalization, with the rapid development of China's economy. Although globalization has brought about a cultural convergence leading to increasing similarity of values (Chiu, 2017), the Chinese management model remains distinctive due to cultural influences. China has had a certain impact on Asian culture as a nation with a long history and a huge population around the world. In particular, the cultures such as Japan, South Korea, Singapore and Malaysia, are all based on Confucianism of Chinese culture (Larson \& Kleiner, 1992). This is probably the main explanation why Asian countries are culturally similar but in contrast to European and American cultures. As the core principle of Confucianism, Zhong-Yong plays a unique role in exploring the disparities between the East and the West (Yuan, 2013), because the prevailing theoretical management paradigm in Western is somewhat ambiguous in explaining the subtle differences in organisational behaviour in Asian countries.

Enterprises are facing the challenge of technological progress. In the context of globalization, innovation is seen as a panacea for improving the competitiveness of enterprises. Organisations need to understand employee behaviour, or their satisfaction, to enable them to achieve productivity, efficiency, and new ideas in the workplace to enhance their corporate competitive advantage and innovation (Jadhav et al., 2017). It is easy to see that there are apparent variations between Asian and Western employees in their innovative behaviours, namely radical innovation and incremental innovation. For instance, there are few companies in Asia like "Apple" and "Tesla", but there are many companies like "Xiaomi" and "Vivo". The main distinction between these two types of businesses is the way they innovate. Take Xiaomi as an example, the company uses the supply chain relationship to integrate resources for reorganisation. It is not a technology-centric innovation, but it is definitely a new pattern and a new way of doing business. In particular, Zhou et al. (2019) indicated that such Oriental companies would gain competitive advantages through compositional capability due to the influence of Zhong-Yong culture. Additionally, most research on employee behaviour of Asian countries focuses on the phenomenon and its impact on the organisation, but lack of research on its root causes (Yuan, 2013). Culture as a potential cause of behaviour and action has been debated for many years. People, however, neglect the cognitive mechanisms in which culture can influence behaviour and consider new ideas at the individual level (Zhou et al., 2019). As a consequence, both the theoretical and practical, there is a need for further exploration of the causes of the phenomenon of employee behaviour in Asia, which is why indigenous research is both desirable and relevant. Zhong-Yong thinking (ZYT) is starting to gain recognition as one of the most meaningful terms of Chinese indigenous research. 
Zhong-Yong is a unique system of metacognitive practical thinking (Yang \& Chiu, 1997) and a thinking style of integrating opinions (Wu \& Lin, 2005), a fundamental Chinese indigenous concept that guides the actions and decisions of Chinese people (Yang et al., 2017). Although ZYT is considered to be typical of Chinese thinking, the awareness of how it can affect the workplace behaviour of employees is minimal. On the one side, people with ZYT can integrate resources effectively, so that their firms' products have a competitive advantage due to their composition capabilities or incremental innovation (Zhou et al., 2019). ZYT, on the other hand, has also been referred to by Chinese people as 'mediocrity'. Chinese people generally regard employees with ZYT to have poor performance and lack of initiative. It is even considered to be contrary to entrepreneurship and innovation because people with mediocrity have less aggressive personality (Chen et al., 2018). The role of ZYT in innovation behaviour was, therefore, heatedly debated. So far, few researchers have extensively evaluated the literature on the impact of ZYT on employees' behaviour and outlining future research directions. The current study will fill this void.

Given these gaps mentioned above, this study aims to provide an overview of the effect of ZYT on employees' behaviour. Accordingly, the objectives of this study are: 1) synthesizing the current literature related to ZTY and employees' behaviour, and 2) highlighting the gaps and suggestions in this area of study for future studies. By addressing ZYT, managers can fully understand Chinese local phenomena from the perspective of thinking style and culture. ZYT as one of Chinese indigenous concept that provides ample theoretical basis to explain the social, business, and individual behavioural impacts of such Chinese phenomena (Li et al., 2016). Besides, exploring ZYT can allow practitioners and academia to understand the gaps between Asian and Western successful business models. The quest for common ground while preserving differences is a demand for the development of cultural diversity. Companies in Asian countries should take advantage of their own culture and avoid the negative side of it to develop their business more efficiently. As a result, rising numbers of scholars are calling attention to the development of indigenous management research, since it is based on a localised concept of local cultural heritage and utilizes their influence on the local environment and regional behaviour to provide a better understanding of culture (Chiu, 2017).

The structure of this study is organized as follows. We start our review by contrasting the difference between Chinese and Western thinking style. Next, we include a thorough description of our systematic review procedure. We are followed by a bibliometric analysis of the extant pieces of literature. Finally, we distil the implications and suggestions for future research. 


\section{Background}

Zhong-Yong is the core of traditional Chinese culture, dating back to the Analects of Confucius. Many researchers consider it to be a central principle in Confucianism. In fact, it includes not only the Taoist view of yin-yang but also the Confucian and Buddhist view of "Zhong". Here "Zhong" is more dynamic and complex, which can also be interpreted as "moderation" or "degree" (Hu et al., 2012). People with ZYT are able to find equilibrium and harmony in a dynamic circumstance. Some studies suggest that the concept of yin-yang balancing is close to the concept of Zhong-Yong (Li, 2016). Li (2016) expounded the concept of yin-yang balancing in terms of asymmetric balancing, curvilinear balancing and transitional balancing. Nevertheless, Li (2019) emphasized that Li (2016)'s asymmetrical balancing differs from the practice and theory of Zhong-Yong. Similar terms exist in the West with "ambidexterity" and "paradox". Likewise, research struggle to clarify the fuzzy thinking concept of Zhong-Yong from above two words. Therefore, Zhong-Yong has its specific meaning and significance, which cannot be precisely replaced by other terms.

Scholars' discussion of the Zhong-Yong initially focused on philosophy. Subsequently, with the growth of indigenous psychology, psychologists conducted thorough researches into Zhong-Yong's psychological framework, such as cognition, or thinking style of Chinese people (Chiu, 2000, Wu \& Lin, 2005, Yang, 2010, Yang and Chiu, 1997). The most representative and early research has come from Yang and Chiu (1997) who define Zhong-Yong as a metacognitive-based practical thinking mechanism that leads Chinese people to decide on an optimal plan of action by thinking when coping with a particular event on a daily basis. Afterwards, Yang (2010) reinterpreted ZYT and divided it into collective cultural thinking and individual psychological thinking, which comprises three stages of thinking: Life philosophy thinking; Individual event processing; and Retrospective. Characteristically, although Yang (2010) and Yang and Chiu (1997) are roughly the same summaries, they provide a strong conceptual foundation for subsequent research. For example, Wu and Lin (2005) take opinion expression as the context and apply the concept of ZYT to the integration of opinion differences. They define it as thinking from various perspectives and then choose to act accordingly, taking into account the larger picture. Not only does this definition emphasizes the "Quan" (holistic thinking), "Zhong" (integrative thinking), but it also embodies the characteristics of "He" (harmony).

In cross-cultural studies, holistic thinking, fuzzy thinking, and the indirect thinking style characteristic of Chinese people are found to be markedly different to Western abstract thinking, binary thinking, and direct thinking styles (Spencer-Rodgers \& Peng, 2017). Studies have shown that Chinese thinking is predominantly dialectical and holistic in nature (Spencer-Rodgers and Peng, 2017). Dialectical thinking prioritizes moderate and reasonable neutralizing behaviours (Nisbett et al., 2001). Additionally, Chinese holistic thinking is contrasted with the Western Aristotelian binary logic. Western societies seek the truth, whereas the Chinese do not focus on this concept and are more concerned with taking a holistic perspective and ensuring plurality (Yuan, 2013). The above Chinese thinking does reflect the differences between Chinese and Western thinking, but none of the thinking abstractly covers all characteristics of Chinese thinking. ZYT has all the hallmarks of these features of 
conventional Chinese thinking, such as holistic, indirect, and fuzzy thinking, and embodies traditional Chinese philosophies of Confucianism, Buddhism, and Taoism (Yuan, 2013). Thus, the Chinese people are profoundly affected by ZYT, irrespective of their social, political, or personal backgrounds.

\section{Research Method}

This study is based on a method of a systematic review to analyse and classify the current literature on the topic to understand the influence of ZYT on employees' behaviour in the workplace. The reason why we embrace this methodology is that a systematic review is an approach which relies strictly on a series of scientific processes that identifying, assembling, appraising and synthesizing all the existing relevant literature in a rigorous, transparent, and reproducible manner of drawing reliable conclusions (Daigneault et al., 2014; Petticrew \& Roberts, 2008; Snyder, 2019; Tranfield et al., 2003; Vrontis \& Christofi, 2019). In contrast with the traditional review, systematic review facilitates help to improve clarity, validity, and audibility (Booth et al., 2016b). Although systematic reviews began in the medical science field, more and more recent studies have published related to management that use the systematic review to enhance the consistency of the review process and reduce the likelihood of bias (Tranfield et al., 2003; Vrontis \& Christofi, 2019). All in all, systematic review is the most appropriate methodology for our research purpose, a way of synthesizing the literature to illustrate the effect of ZYT on employees' behaviour.

We adapted the guidelines recommendations for a systematic review from Wang and Ngai (2020). These steps include: 1) designing the review; 2) screening and selection of studies; and 3) data synthesis and analysis, including initial data analysis and bibliometric analysis. In the following section, we detailly describe each step of the procedure.

\section{Designing the Review}

\section{Identifying Keywords and Search Strategy}

To classify all studies within the scope of ZYT, we conduct preliminary literature search using the terms "Zhong Yong" in the several potential bibliographic databases. In order to fulfil the comprehensive approach of the review, we choose three databases for studies published that include two well-established databases, and most are in English (i.e., Web of Science and Scopus) and Google scholar (Chinese). The reason for choosing the Chinese language is to avoid limiting the function of databases, which could protect the review from being affected by potential language bias (Booth et al., 2016b). Most notably, China has been the second-larger country in terms of the number of articles published in the Web of Science (WOS) (National Science Board, 2018). However, in China, research activities are not adequately represented by WOS, with more than $97 \%$ of Chinese academic journals excluded (ISTIC, 2014). Although the number of Chinese researchers writing in English has increased, some Chinese scholars prefer to publish in Chinese-dominated journals (Shu et al., 2019). ZYT as unique thinking style in China. We assume that most of the studies have been published in the Chinese language. 
After determining the keywords and bibliographic database, we identified that the search strategy with three-stage. First of all, undertaking scoping search in above databased. The terms "Zhong Yong" is used in the search string to represent ZYT. Secondly, using a search filter to limit the scope of research to management and exclude patent and citation where the database has the corresponding search filtering function. This search strategy yielded an initial sample of 167 related studies.

\section{Screening and Selection of Studies}

In the screening and selection phase, we considered determining which studies to include. Firstly, since we have searched several databases, 15 duplicate studies were identified and removed from the preliminary search results, resulting in 142 studies. Secondly, after screening the title and abstract of the studies, we excluded 125 studies and obtained 17 studies, including four in English and 13 in Chinese. Thirdly, we have developed the content of exclusion criteria, which are described in the following sections.

\section{Inclusion Criteria}

Aligned with Dada (2018) and Vrontis and Christofi (2019), three categories of inclusion criteria were used to assess the studies included in the systematic review. They are, 1) determining the review boundaries; 2) identifying the keywords and search strings, and; 3) specifying the timeframe of the studies. First, the search boundaries were set as bibliographic databases (Vrontis \& Christofi, 2019), resulting in three databases have been aforementioned. Moreover, in order to provide a holistic review of the subject, studies' language set as published in both English and Chinese, but Chinese articles required have English title and abstract. Second, the keywords and strings have been discussed in the previous section. Third, to better capture all related studies, we have not set a precise timeline, but October 2019 marks the end of this literature quest.

\section{Exclusion Criteria}

An additional evaluation of 17 articles was performed based on various exclusion criteria. First, since this review is aimed at ZYT, some studies are in the field of psychology and education, therefore, we excluded the studies that are not related to management. Second, we have excluded the studies that do are non-related to thinking style boundaries, such as studies on Chinese Zhong-Yong culture and Zhong-Yong philosophy. Third, to arrive at comprehensive data syntheses and analyses, we excluded non-full text articles. Fourth, we are focused on the studies published in peer-review academic journals, thus excluding non-academic articles, such as the books, book chapters, book reviews, thesis, discussion papers, working papers, editorials, and conference papers, in a similar way to Gast et al. (2017) and Vrontis and Christofi (2019). 


\section{Quality Assessment}

Quality assessment, also known as critical appraisal, is designed to systematically consider the validity of the selected individual studies and their relevance to this systematic review based on research questions (Boland et al., 2017; Booth et al., 2016a; Parkes et al., 2001). In this phase, we ensure that selected English studies are published in the peer-reviewed journals indexed in ISI (Ahmad and Omar, 2016), while Chinese studies are indexed on Chinese Social Science Citation Index (CSSCI) ( $\mathrm{Li}$ and $\mathrm{Lu}, 2016$ ).

\section{Further Search Processes}

According to Vrontis and Christofi (2019), we manually conducting bibliographic searched the reference list for all selected studies, which increased two studies. Next, to ensure that no articles are missing, we presented a list of studies to two experts in the field and consulted them to provide us with studies that our process was not included. This last step added three more Chinese studies. After the inclusion, exclusion, and quality assessment criteria for the retrieved studies, we retrieved 22 articles published in peer-reviewed journals, including four in English and 18 in Chinese. Figure 1 shows the procedure for selecting articles for this review.

\section{Figure 1: Procedure for Selecting Studies}

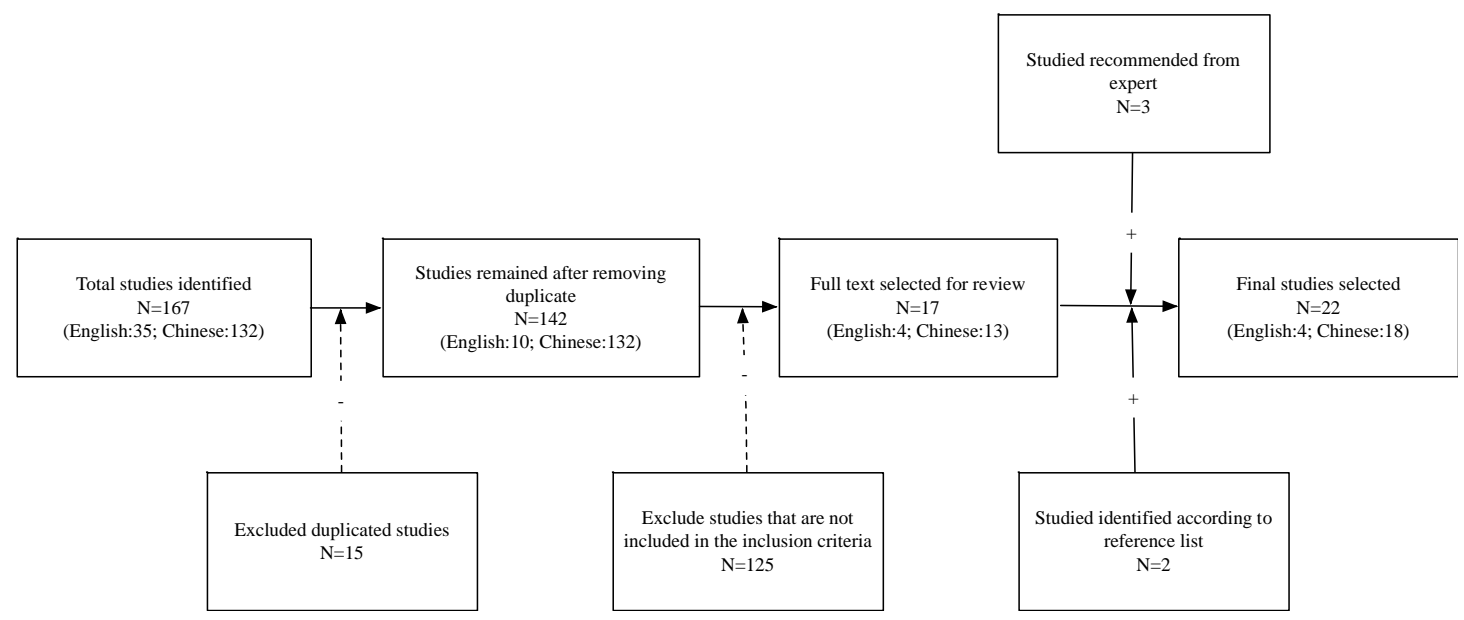

\section{Data Synthesis and Analysis}

This stage includes initial data analysis and bibliometric analysis. A coding sheet was developed of initial data analysis according to previous reviews ( $\mathrm{Li} \& \mathrm{Lu}, 2016$; Ortiz-Avram et al., 2018). The coding sheet contains several parameters such as year of publication, authorship, and measurements. The bibliometric analysis focuses on to identify the cluster of the literature based on keywords findings. 


\section{Data Analysis}

\section{Initial Data Analysis}

\section{Year of Publication, Number of Articles published in Journals}

A total of four English (18\% of the total articles) and 18 Chinese articles (82\% of the total articles) were taken into the consideration set. The trend in the number of articles published annually is shown in Figure 2. As of 2010, when English $(n=1)$ articles appeared, the impact of ZYT on employees' behaviour has been the focus. The number of articles has risen substantially, peaking in $2014(\mathrm{n}=5)$. In particular, among the retrieved articles, most of English articles $(n=3)$ were published after 2014, accounting for more than half (75\% of total English articles). With regards to Chinese articles, $67 \%$ of all Chinese publications have been published after $2014(n=12)$. In general, the number of Chinese and English articles published in the past five years accounted for $68 \%$ of the total number of publications $(n=15)$, which indicates that research in this field has attracted the attention of academics in recent years.

Figure 2: Year of Publication

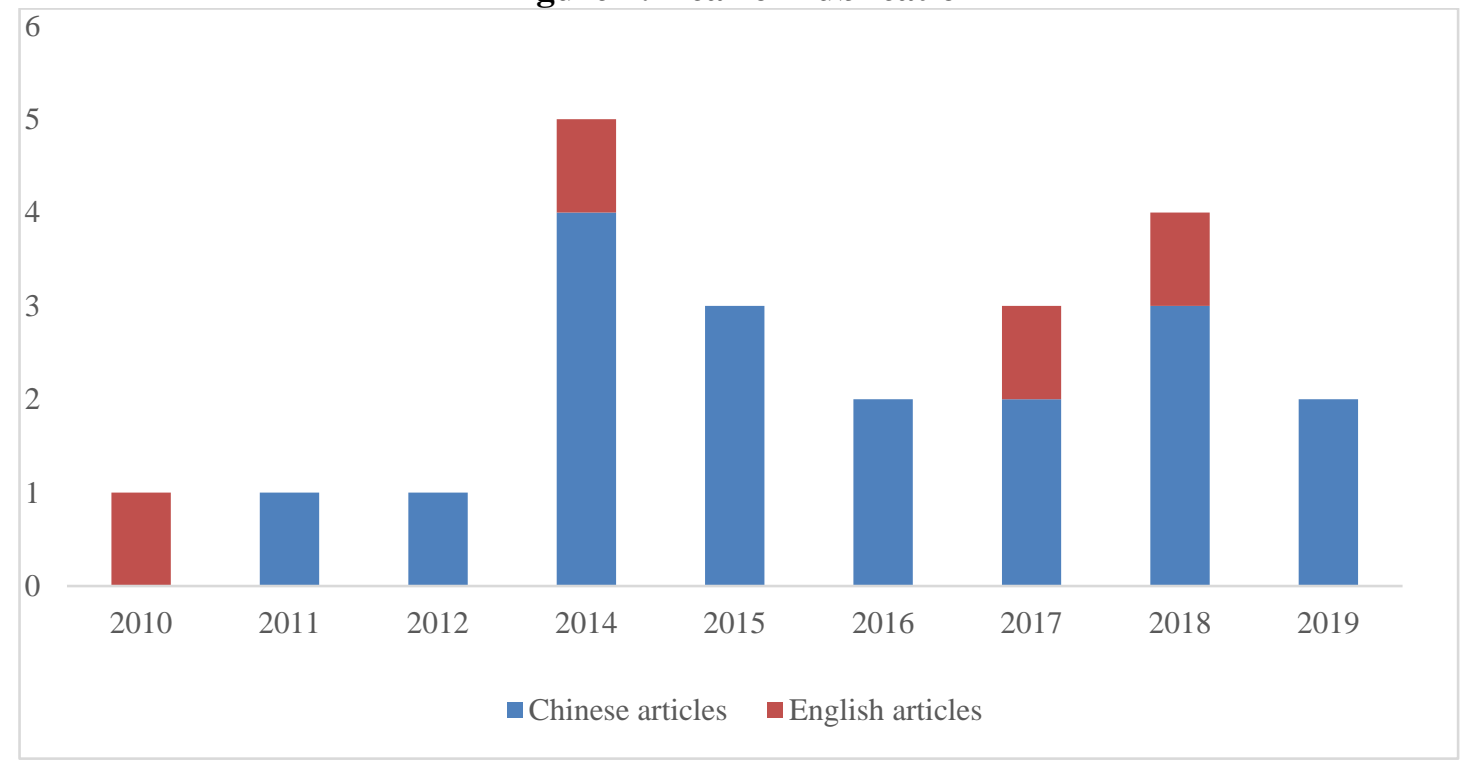

The source journal of the literature is an essential way for scholars to publish their results. The retrieved articles were published in 17 various peer-reviewed journals, including Three English Journals and 14 Chinese Journals (Figure 3). We identified that three of these journals published more than one article in this field, two of which were in Chinese journals, and only one was in English journals. Among the Chinese journals, Acta Psychologica Sinica $(n=4)$ dominated the platform for the relevant field of study, followed by Economic Management in China $(\mathrm{n}=2)$. Among the English journals were Asian Journal of Social Psychology $(\mathrm{n}=2)$ with the highest number of articles, along with Chinese Management Studies $(\mathrm{n}=1)$ and Management Organization Review ( $\mathrm{n}=1)$. Briefly, Acta Psychologica Sinica $(\mathrm{n}=4,22 \%$ of total Chinese publications) and Asian Journal of Social Psychology ( $\mathrm{n}=2,50 \%$ of total English publications) were the two journals that published the most articles in this area in which psychology is their common field. 
Figure 3: List of Journals

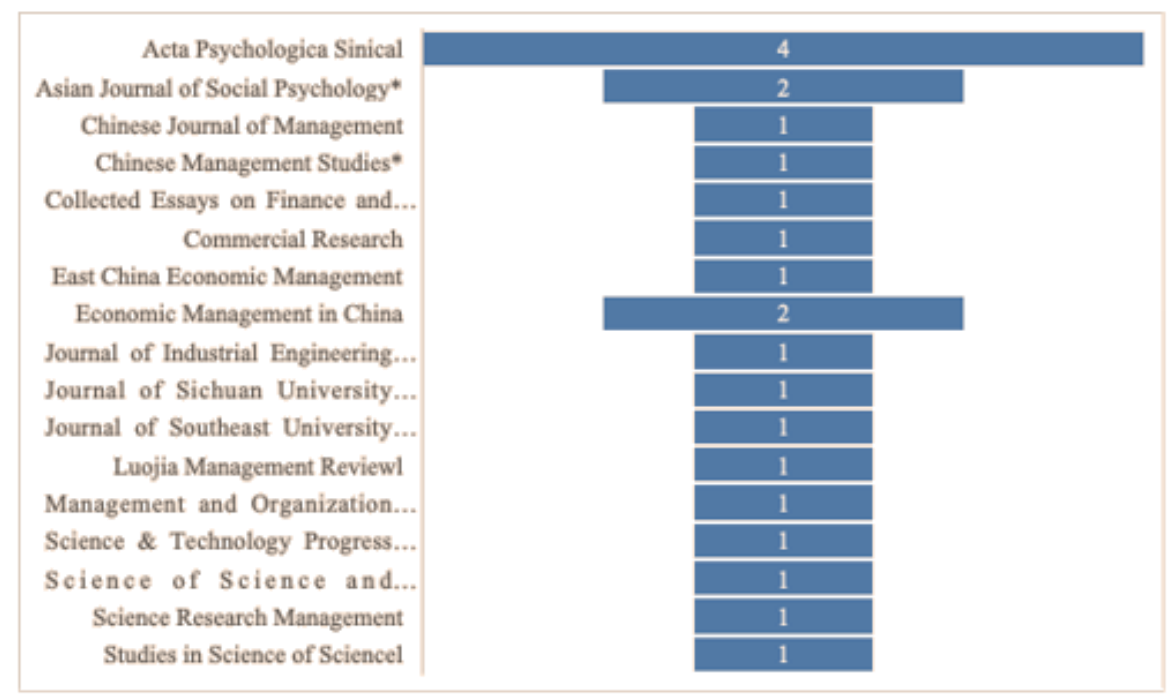

\section{Authorship}

The authorship analysis of reviewed articles was developed based on a systematic review study by Vrontis and Christofi (2019). To start with, we identified 13 authors contributes to this research domain from six institutions in China with English publications. From statistical data, articles published in English journals in this field are completed by the cooperation of authors. 50\% of English articles are completed by three or more authors $(n=2), 50 \%$ of them are from the same institution $(n=2)$, and all other institutions are based in China $(n=4)$. Surprisingly, in addition to mainland China, scholars from Macau China, and Taiwan China have also joined this research field.

Continuing, among Chinese publications, 42 authors have sought to determine ZYT in the area. $72 \%$ of Chinese articles came from the same institution $(n=13) ; 94 \%$ of them were published by more than two authors $(n=17)$. Considering the research institutions, the institutions that have participated in the publication of English journals are all diverse. Published in Chinese journals, the most involved was Wuhan University $(n=4)$ in China. For authors, most authors have published only one article in this field. Notably, author Du Jing has published the most in this field, with a total of four Chinese publications. 
Table 1: Authorship Analysis

\begin{tabular}{|c|c|c|}
\hline Authorship Characteristics & \multicolumn{1}{c|}{$\begin{array}{c}\text { No. of English } \\
\text { Publications } \\
\text { Number of authors }\end{array}$} & No. of Chinese Publications \\
\hline \multicolumn{3}{|c|}{0} \\
\hline One & 2 & 1 \\
\hline Two & 2 & 8 \\
\hline Three or more & Number of Countries \\
\hline One & 4 & 18 \\
\hline Two & 0 & 0 \\
\hline Three or more & 0 & 0 \\
\hline \multicolumn{2}{|c|}{} \\
\hline One & Number of Institutions \\
\hline Two & 2 & 13 \\
\hline Three or more & 2 & 2 \\
\hline
\end{tabular}

\section{Measurements of Zhong-Yong Thinking in Survey}

In our consideration set, 22 reviewed articles used a survey to investigate the effect of ZYT on employees' behaviour. We identified five questionnaires scales to measure ZYT. Among the five scales, three were developed from Taiwanese, one from Hong Kong, and one from mainland Chinese. In fact, these five scales were all developed on the basis of the scale of Yang and Chiu (1997), with a different emphasis: Yang and Chiu (1997) suggested that the system of ZYT is a set of practical thinking, which contains 16 items of 8 dimensions, namely, observing changes, valuing harmony, thinking at two levels, putting the overall situation first, being reasonable, retreating for progress, paying attention to consequences, and not going to extremes; Chiu (2000) focuses on the level of concrete actions with 14 items in three dimensions, including the target of actions, the mode of perception and the execution of actions; $\mathrm{Wu}$ and Lin (2005) emphasizes on the thinking mode of integrating opinions, including 13 items in three dimensions: holistic thinking, perspective integration and harmonious maintenance. Huang et al. (2012) developed nine items with the transformability of overall thinking. Du and Yao (2015) explored eight items of individual constructs from the perspective of their interaction with the external environment.

It can be concluded that, first, the scale developed by Wu and Lin (2005) that takes an expression of opinions as situational narration is the most popular scale in the field of management and is adopted by both Chinese and English articles. Second, the scale developed for the Chinese mainland was not adopted by English articles, but a few of Chinese articles were studied based on this scale. Finally, the research objects of the development of these scales are university students except for the scales of Du and Yao (2015), which are discussed by middle managers. 
Table 2: Measurements of Zhong-Yong Thinking

\begin{tabular}{|c|c|c|c|}
\hline Measurement & International publications & Chinese publications & Total \\
\hline $\begin{array}{l}\text { Wu and Lin } \\
(2005)\end{array}$ & Pan and Sun (2018) & $\begin{array}{l}\text { Cai and Geng (2016); Du et al. } \\
\text { (2014); Duan and Ling (2011); Hu } \\
\text { et al. (2012); (Liao and Dong, } \\
\text { 2015); Ma and Cai (2018); Qing } \\
\text { and Liu (2014); Shen et al. (2019); } \\
\text { Sun et al. (2014); Wei (2019); } \\
\text { Yang et al. (2017); Zhang and } \\
\text { Long (2016); Zhang and Gu } \\
\text { (2015); (Zhou et al., 2017) }\end{array}$ & 15 \\
\hline $\begin{array}{l}\text { Du and Yao } \\
(2015)\end{array}$ & & $\begin{array}{l}\text { Du and Duan (2017); (Du et al., } \\
\text { 2018); Du and Yao (2015) }\end{array}$ & 3 \\
\hline Chiu (2000) & $\begin{array}{l}\text { Qu et al. (2018); Yao et al. } \\
(2010)\end{array}$ & & 2 \\
\hline $\begin{array}{l}\text { Yang and Zhao } \\
\text { (1997) }\end{array}$ & & Yao and Fan (2014) & 1 \\
\hline $\begin{array}{l}\text { Huang, Lin and } \\
\text { Yang (2012) }\end{array}$ & Chou et al. (2014) & & 1 \\
\hline
\end{tabular}

\section{Bibliometric Analysis}

Along with the fragmentation of topics in academic publications, bibliometric analysis is becoming increasingly essential to the academic community due to its ability to classify prominent research and clusters of literature on a particular topic or area of interest (Wang \& Ngai, 2020). Application of VOS viewer tools can help the review visualize by evaluating keywords occurrence and explore the cluster of the research fields involved (Vallaster et al., 2019). Table 3 displays the list of most occurrence keywords analysed from the VOS viewer. As can be seen from the table, employee behaviour of innovation, voice, creativity, and citizenship have frequently been addressed in this field. Since the related fields do not have abundant data, one keyword used as the minimum criterion to analysis and obtain 61 items in total. The relationships between the keywords can be divided into three major clusters, namely innovation behaviour, voice behaviour, and employee performance, according to the graph shown in Figure 4.

Table 3: Occurrence of Keywords

\begin{tabular}{|l|l|l|l|}
\hline No. & Keywords & Occurrences & Total link strength \\
\hline 1 & Zhong-Yong thinking & 22 & 81 \\
\hline 2 & Innovation behaviour & 9 & 31 \\
\hline 3 & Voice behaviour & 6 & 23 \\
\hline 4 & Creativity & 3 & 11 \\
\hline 5 & Employee performance & 3 & 13 \\
\hline 6 & Citizenship behaviour & 2 & 9 \\
\hline 7 & Incremental innovation & 2 & 8 \\
\hline 8 & Organisational harmony & 2 & 5 \\
\hline 9 & Team-member exchange & 2 & 6 \\
\hline
\end{tabular}


Figure 4: Co-occurrence of Keywords

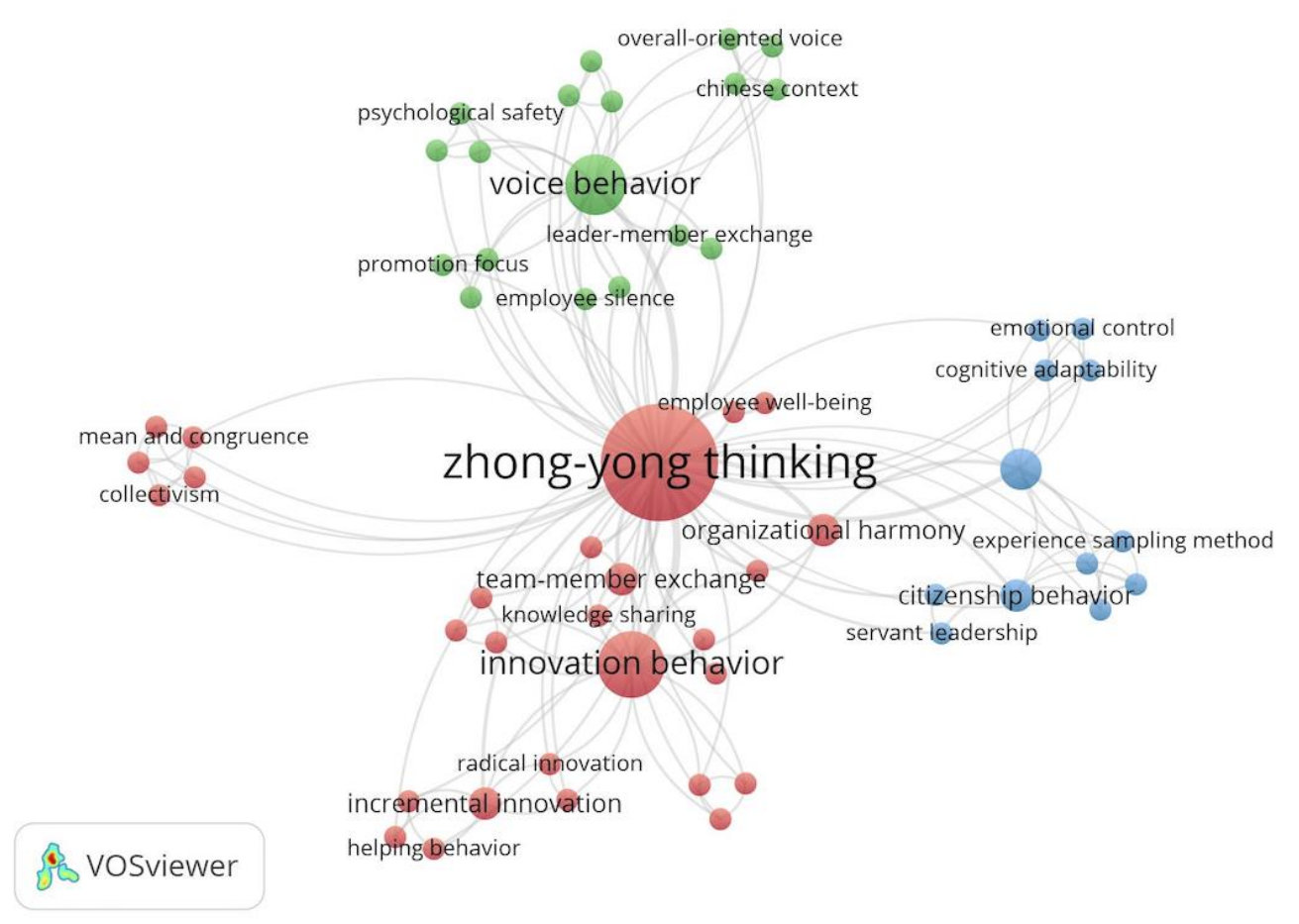

Cluster One: Zhong-Yong Thinking and Innovation Behaviour

In this cluster, scholars mainly have the following views: some of them suggest that ZYT may have directly (Liao \& Dong, 2015) or indirectly (Du \& Duan, 2017; Zhang and $\mathrm{Gu}, 2015)$ positive impact on innovation behaviour; Some of them argue that ZYT has a various influence on different types of innovation (Du \& Duan, 2017; Du et al., 2018); Others state that there is a non-linear relationship between ZYT and innovation (Wei, 2019). Specifically, researchers who favour positive influence argue that employees with high levels of ZYT tend to cooperate. The greater the cooperation between individuals, the easier it is for knowledge to be shared and combined (Chen et al., 2018; Wei, 2019), and the greater the likelihood of innovative behaviours being exhibited (Liao \& Dong, 2015). Moreover, scholars stated that ZYT has a strong context-contingent (e.g. high innovative cognition; and team colleague negative pressure) effect on the innovative behaviour of employees (Du et al., 2014). For instance, organisational harmony, the atmosphere of Zhong-Yong and the atmosphere of error management all influence the impact of ZYT on innovation among employees (Du et al., 2018; Zhou et al., 2017; Liao \& Dong, 2015). Remarkably, Wei (2019) claims that ZYT, as a type of thinking style, has the status of moderate ZYT or excessive ZYT; the author puts forward a non-linear view that ZYT has an inverted $u$ shaped influence on innovative behaviour; in other words, moderate ZYT can promote innovative behaviour, whereas excessive ZYT can inhibit innovation. 


\section{Cluster Two: Zhong-Yong Thinking and Voice Behaviour}

Voice behaviour, as an extra-role behaviour of organisational citizenship, is seen as an initiative behaviour aimed at fostering employees' creativity and innovation and has become the second primary focus of reviewed articles (Cai \& Geng, 2016; Duan \& Ling, 2011; Ma \& Cai, 2018; Qing \& Liu, 2014; Yang et al., 2017). Employees not only pursue and maintain the harmony of interpersonal relationship to have a positive attitude towards the behaviour of helping colleague (Du \& Duan, 2017), but also actively towards the behaviour of overall-oriented voice (Duan \& Ling, 2011) and harmony voice (Qu et al., 2018). Employees with high ZYT are more vigilant about their relationship with their superiors, accordingly, they are not willing to make more voice behaviour due to the exchange of high-quality superior-subordinate guanxi. Hence, Qing and Liu (2014) argue that the higher ZYT of employees, the weaker the positive relationship between superior-subordinate guanxi and voice behaviour.

\section{Cluster Three: Zhong-Yong Thinking and Employees' Performance}

In this cluster, citizenship behaviour, counterproductive work behaviour, and helping behaviour are all closely linked to employee performance. One emphasizes on the level of individual analysis category ( $\mathrm{Hu}$ et al., 2012) and the other two focuses on multiple levels of analysis (Jiang et al., 2016; Pan \& Sun, 2018). The impact outcomes of all these three-studies are related to employee performance. By using paired questionnaires of 361 employees in 62 teams from Chinese organisations, Pan and Sun (2018) did not successfully test the direct effect of ZYT on employee adaptive performance, but proposed the indirect effect of them via cognitive adaptability and emotional control with a moderator of job complexity. The relationship between ZYT and cognitive adaptability, emotional is more substantial when an employee at a higher level of job complexity (Pan \& Sun, 2018). Similarly, Jiang et al. (2016) also argued the indirect impact of ZYT on employee performance by a team level construct, leader-member exchange. On the other hand, $\mathrm{Hu}$ et al. (2012) proposed different perspectives. The authors not only verified the direct positive impact of ZYT on employee performance but also suggested that the harmony of ZYT can keep the harmonious atmosphere in the organisation, thereby improving employee performance.

\section{Discussion}

In this section, we discuss the potential research direction related to the impact of ZYT on employees' behaviour based on the data analysis of this systematic review. Table 4 summarizes the future research directions mentioned in the selected literature. Through this systematic review, we have found that the study in the field of ZYT and employees' behaviour is still in its infancy. Exploring the effect of various cultural thinking style on employees' behaviour is worthy of researchers' attention.

To start with, although the study of Zhong-Yong has been defined and measured the basis of the discussion of ancient documents, it is still controversial among scholars. Yang (2010) argued that the study of cultural constructs should not be confined to ancient classics. With the development of society, the psychological functioning of contemporary Chinese people will also be different, so that the concept of Zhong- 
Yong may also change to some extent. What is more, understanding of the link between ZYT and innovation remains essential. Some studies argue that ZYT has a positive impact on innovation, despite the fact that, to a certain degree, Chinese society widely regards Zhong-Yong as a negative attribute. It is significant to consider that the findings of the previous studies differ from the general view of ZYT.

Moreover, as a sort of cognitive thinking, ZYT not only expects researchers to be acquainted with management knowledge but also need to understand the cognitive thinking from a psychology perspective. However, most of the existing research concentrates on employees' behaviour excessively, and it is not clear enough how individuals' cognitive thinking is to be clarified. For instance, many articles argued that the concepts of innovation and creativity are identical. Yao et al. (2010) have nevertheless pointed out that creativity occurs before the start of the innovation process and is a kind of mental intelligence. Therefore, we call for future research to explore the role of ZYT from a psychological theory point of view. Additionally, the study of ZYT in conflict management is still blank. Since ZYT is good at solving the contradictions of interpersonal relationships, it is worth noting that how ZYT, with harmony at its core, affects interpersonal conflict at work.

Furthermore, several studies have pointed to the fact that China is so large that the findings of the study can be limited the extent of its limited region (Cai \& Geng, 2016; Chen et al., 2018; Du \& Duan, 2017; Du et al., 2014; Duan \& Ling, 2011; Liao and Dong, 2015; Qing and Liu, 2014; Zhang and Long, 2016). It is possible that regional problems may lead to various levels of ZYT. Stratified sampling can be used in future studies to improve the generalization of the research conclusions. Besides, all previous studies are focused on mainland China, enabling potential studies can compare other regions or countries in an Asian country (Wei, 2019). In terms of data collection, future research can follow multi-source methods to collect data (Sun et al., 2014; Zhang \& Gu, 2015), such as integrate with self-evaluation and other evaluation. Although this method has been used in some of the prior researches, it still has reference value in future researches.

In general, we call upon researchers to recognize the value of using indigenous studies to address better and comprehend practical issues. For example, the Covid-19 pandemic has shown that the behaviour of individuals in various nations can be distinguished by distinctive local characteristics. We hope that future research will focus on such issues, for example, the role of ZYT in crisis management and employee well-being. 
Table 4: Future Directions Proposed by Previous Literature

\begin{tabular}{|l|l|}
\hline \multicolumn{1}{|c|}{ Future Directions } & \multicolumn{1}{|c|}{ Citations } \\
\hline $\begin{array}{l}\text { Employ longitudinal designs to investigate causality } \\
\text { between variables. }\end{array}$ & $\begin{array}{l}\text { Cai and Geng (2016); Chou } \\
\text { (2014); Du and Yao (2015); } \\
\text { Duan and Ling (2011); Hu } \\
\text { et al. (2012); Luo et al. } \\
\text { (2018); Ma et al. (2017); Ma } \\
\text { et al. (2018); Wei (2019); } \\
\text { Yao et al. (2010); Zhang and } \\
\text { Long (2016) }\end{array}$ \\
\hline $\begin{array}{l}\text { Some of the studies have a common method bias so that a a } \\
\text { multi-source data collection method can be adopted. }\end{array}$ & $\begin{array}{l}\text { Sun et al. (2014); Zhang and } \\
\text { Gu (2015) }\end{array}$ \\
\hline $\begin{array}{l}\text { Select the research objects by the method of stratified } \\
\text { sampling. }\end{array}$ & $\begin{array}{l}\text { Cai and Geng (2016); Chen } \\
\text { et al. (2018); Du and Duan } \\
\text { (2017); Du et al. (2014); } \\
\text { Duan and Ling (2011); Qing } \\
\text { and Liu (2014); Zhang and } \\
\text { Long (2016) }\end{array}$ \\
\hline $\begin{array}{l}\text { Compare the influence of ZYT of employees from Hong } \\
\text { Kong, Macao, Taiwan and mainland China on innovation. }\end{array}$ & Wei (2019) \\
\hline $\begin{array}{l}\text { To explore the moderating effect of ZYT in different } \\
\text { dimensions. }\end{array}$ & Yao and Fan (2014) \\
\hline $\begin{array}{l}\text { Explore ZYT in employees faces a situation of uncertainty, } \\
\text { or ethical dilemma, how to adjust their emotion, for } \\
\text { example, abusive supervisor. }\end{array}$ & Chou et al. (2014) \\
\hline $\begin{array}{l}\text { Examine whether there are some organisational strategies or } \\
\text { policies for improving the transition from creativity to } \\
\text { innovative behaviour for the employee with high ZYT. }\end{array}$ & Yao et al. (2010) \\
\hline $\begin{array}{l}\text { Explore the impact of ZYT on organisational performance } \\
\text { based on interpersonal relationships. }\end{array}$ & Chen et al. (2010) \\
\hline $\begin{array}{l}\text { Examine whether ZYT applies to non-Confucianism } \\
\text { culture. }\end{array}$ & $\begin{array}{l}\text { Chou et al. (2014); Ma et al. } \\
\text { (2018) }\end{array}$ \\
$\begin{array}{l}\text { Consider the difference in ZYT of different roles. (2017); Zhang } \\
\text { and Gu (2015) }\end{array}$ \\
\hline
\end{tabular}

\section{Conclusion}

This study provides valuable insight into the impact of ZYT on employees' behaviour, enabling future researchers to gain a better understanding of the role of ZYT as identified in quantitative research. Based on the bibliometric analysis, gaps in this field have been discussed with the aim of highlight multiple future research directions to enrich the literature.

Along with this study's theoretical implication, we provide useful guidance for practitioners by presenting the literature on ZYT and employees' behaviour. In particular, as far as the organisation is concerned, making progress through innovation is the common goal of the enterprise. As a result, employees with ZYT in line with the organisational environment have not opposed innovation (Du et al., 2018), but are not favoured by radical innovation. However, incremental innovation, as a form of modest and moderate innovation, is a series of small improvements or upgrades made 
by enterprises to their existing resources or technologies, which conforms to the characteristics of ZYT, which emphasizes balance and harmony (Du et al., 2018). Additionally, scholars have not found that ZYT has a direct impact on incremental innovation (Du \& Duan, 2017; Du et al., 2018). Du and Duan (2017) and Zhang and $\mathrm{Gu}$ (2015) both agree that employees' occupation psychological factors (e.g. environmental mastery; job satisfaction) provides a significant foundation for employee innovation, and plays an overall mediating role in the positive relationship between ZYT and incremental innovation. Consequently, the practitioner should consider the policies to enhance employees' job satisfaction in order to boost their incremental innovation behaviour (Ye et al., 2020b).

\section{Practical Implications for Asian Business}

ZYT, as a way of thinking, has a profound influence not only on the Chinese people now, but will also influence future generations of ethnically Chinese people in other Asian countries. People of Chinese descent in other Asian countries typically represent an important and prosperous part of those countries' communities. ZYT is a culturally based, nuanced way of thinking that incorporates all facets of Chinese people's work and life, including human affairs. As China engages more closely with the economies and societies of Asian countries, the role of ZYT in the Asian business economy is worth exploring in greater depth.

The innovations brought about by ZYT fit more for East Asian countries. East Asian countries are not as capable of radical innovation as are Western ones but are more capable of incremental innovation. ZYT provides a model of incremental innovation, which is more readily accepted by current East Asian societies, even without fundamental reform. The integrated, harmonious existence of ZYT, which is more readily embraced by Asian communities, makes the employees in East Asian countries more prone to incremental innovation than to the radical innovation that is common in the West. In East Asia, employees with ZYT can use their strengths to consolidate resources under their current circumstances, gradually reducing the disparities with advanced developed Western economies, and establish a complementary role in innovation.

Moreover, ZYT emphasizes harmony and lack of conflict, which can contribute to a more stable business. Cooperative working is often difficult for employees of various ethnic groups in Asian countries, particularly if their workplaces are multi-ethnic as different groups can struggle to understand each other, and conflicts and inefficiency can arise. Because employees with ZYT get along well with their colleagues of all ethnic groups, they not only mitigate the business costs that result from conflict between employees but also foster the development and competitive advantage of Asian corporate culture. For instance, in the context of increasing integration of countries in the Association of Southeast Asian Nations (ASEAN), people's mobility is growing. Companies in the ASEAN are starting to recruit people from different countries and nationalities to work together, and ZYT can effectively integrate all types of people. 
Voice behaviour can improve the functions of an organisation. However, the voice of workers in Asian countries and the equality of dialogue with superiors are generally lacking. This is partly due to the fact that less skilled employees are hesitant to talk to those with whom they are not well acquainted. Because of the holistic nature of ZYT, when employees are confronted with a problem, they will consider it from all angles. Although voice behaviour relates to the overall interests of the company, employees who express their voice authentically may cause unnecessary difficulties due to the high-power distancing in Asian culture. Consequently, employees with ZYT do not incline to exercise voice behaviour. It is therefore in the best interests of the employee to say as little as possible or to respond to the needs of his superiors from the point of view of the employee only, without considering the company's broader interests.

In most Asian small and medium-sized enterprises, the upper and lower levels frequently lack a mechanism for direct dialogue (Ye et al., 2020a). For example, middle management often acts as a means of communicating the thoughts and strategies of top management to the lower level of employees. In this circumstance, employees cannot articulate their voices easily and authentically, which causes not only an issue within the hierarchy but above all, a misalignment of information. The lack of understanding of superiors and subordinates also leads to inefficiency and mistakes in decision-making. Thus, ZYT features can be crucially employed to establish policies to improve employee voice behaviour and facilitate a seamless flow of information within the company.

In short, in an Asian culture influenced by Confucianism, and especially in an open society, the influence of ZYT on employees cannot be ignored. Practitioners should develop strategies for encouraging the positive effects of ZYT, such as increased creativity, and reducing the adverse effects of ZYT, such as diminished voice behaviour.

\section{References}

Ahmad, S., \& Omar, R., (2016), "Basic corporate governance models: A systematic review", International Journal of Law and Management, vol. 58, no. 1, pp. 73-107.

Boland, A., Cherry, G., \& Dickson, R., (2017), Quality assessment: Where do I begin? Doing a systematic review: A student's guide, Sage Publication, London.

Booth A., Sutton, A., \& Papaioannou, D., (2016a), Assessing the evidence base: Systematic approaches to a successful literature review, Sage Publication, London.

Booth, A., Sutton, A., \& Papaioannou, D., (2016b), Systematic approaches to a successful literature review, Sage Publication, London.

Cai, X., \& Geng, X., (2016), "Self-protective implicit voice belief and employee silence: A research in the context of China", Science of Science and Management of S. \& T., vol. 37, no. 10, pp. 153-163.

Chen, J., Lin, Y., \& Liu, S., (2010), "Study on the relationship between top leaders' goldenmean thinking and organizational performance: Mechanism and contingency", Nankai Business Review, vol. 2, pp. 132-141.

Chen, Y., Chen, Z., \& Jiang, B., (2017), "Can Zhong-Yong thinking improve the decision effectiveness of entrepreneurial teams? - The mediating role of behavioural integration", Scientific Decision Making, vol. 7, pp. 85-104.

Chen, Y., Qi, Z., \& Chen, Z., (2018), "An analysis of the effect and mechanism of ZhongYong thinking on team innovation”, Forecasting, vol. 37, no. 2, pp. 15-21. 
Chiu, C., (2000), "Assessment of Zhong-Yong (dialectic) thinking: Preliminary findings from a cross-regional study", Hong Kong Journal of Social Science, vol. 18, pp. 33-35.

Chiu, C. Y., (2017), "Culture matters: A perspective advancing cross-cultural and indigenous research", Management and Organization Review, vol. 13, no. 4, pp. 695-701.

Chou, L. F., Chu, C. C., \& Yeh, H. C., (2014), "Work stress and employee well-being: The critical role of Zhong-Yong", Asian Journal of Social Psychology, vol. 17, no. 2, pp. $115-127$.

Dada, O., (2018), "A model of entrepreneurial autonomy in franchised outlets: A systematic review of the empirical evidence", International Journal of Management Reviews vol. 20, no. 2, pp. 206-226.

Daigneault, P. M., Jacob, S., \& Ouimet, M., (2014), "Using systematic review methods within a Ph.D. dissertation in political science: Challenges and lessons learned from practice", International Journal of Social Research Methodology, vol. 17, no. 3, pp. 267-283.

Du, J., \& Duan, C., (2017), “The mechanism of Zhong-Yong's effect on individuals: Taskand relation-oriented perspectives", Luojia Management Review, vol. 1, pp. 77-90.

Du, J., Qiu, Y., \& Ying, J., (2018), "Does Zhong Yong hamper creativity? An empirical multi-level study", Studies in Science of Science, vol. 36, no. 2, pp. 378-384.

Du, J., Ran, M., \& Cao, P., (2014), "Context-contingent effect of Zhong Yong on employee innovation behaviour", Acta Psychologica Sinica, vol. 46, no. 1, pp. 113-124.

Du, J., \& Yao, J., (2015), "Zhong Yong: The connotation and its relationship with collectivism", Chinese Journal of Management, vol. 12, no. 5, p. 638.

Duan, J., \& Ling, B., (2011), "A Chinese indigenous study of the construct of employee voice behaviour and the influence of Zhong Yong on it", Acta Psychologica Sinica, vol. 43, no. 10 , pp. 1185-1197.

Gast, J., Gundolf, K., \& Cesinger, B., (2017), "Doing business in a green way: A systematic review of the ecological sustainability entrepreneurship literature and future research directions", Journal of Cleaner Production, vol. 147, pp. 44-56.

Huang, C., Lin, Y., \& Yang, C., (2012), "Revision of the Zhong Yong belief value scale", Indigenous Psychological Research in Chinese Societies, vol. 38, pp. 3-41.

Jadhav, V., Seetharaman, A., \& Rai, S., (2017), "Employee expectation to demonstrate innovative work behaviour in Asia", The Journal of Asian Finance, Economics and Business, vol. 4, no. 1, pp. 67-78.

Jiang, W., Jia, L., \& Liu, D., (2016), "Leader member exchange: The role of middle way thinking and high commitment work system", Luojia Management Review, vol. 1, pp. $1-17$.

Larson, H. H., \& Kleiner, B. H., (1992), "Understanding and effectively managing Asian employees", Equal Opportunities International, vol. 11, no. 2, pp. 18-23.

Li, J., \& Lu, Y., (2016), "Chinese culture in tourist research: A review and comparison of Chinese and English studies in 1993-2012", Tourism Review, vol. 71, no. 2, pp. 118134.

Li, P. P., (2016), "Global implications of the indigenous epistemological system from the East", Cross Cultural \& Strategic Management, vol. 23, no. 1, pp. 42-77.

Li, P. P., Sekiguchi, T., \& Zhou, K., (2016), The emerging research on indigenous management in Asia, Springer, London.

Li, X., Andersen, T. J., \& Hallin, C. A., (2019), "A Zhong-Yong perspective on balancing the top-down and bottom-up processes in strategy-making", Cross Cultural and Strategic Management, vol. 26, no. 3, pp. 313-336.

Liao, B., \& Dong, W., (2015), "The study for the relationship among golden-mean thinking, organizational harmony and innovation behaviour of knowledge staff", Science \& Technology Progress and Policy, vol. 32, no. 7, pp. 150-154.

Luo, J., Yi, M., \& Zhong, J., (2018), "Effects of ambidextrous leadership on prosocial silence", Journal of Management Science, vol. 2, pp. 105-119.

Ma, C., Gu, J., \& Liu, H., (2017), "Entrepreneurs' passion and new venture performance in China", International Entrepreneurship and Management Journal, vol. 13, no. 4, pp. 1043-1068. 
Ma, C., Liu, H., \& Gu, J., (2018), "How entrepreneurs' Zhong Yong thinking improves new venture performance: The mediating role of guanxi and the moderating role of environmental turbulence", Chinese Management Studies, vol. 12, no. 2, pp. 323-345.

Ma, P., \& Cai, S., (2018), "A cross-level analysis of motivation internalization of the effect of paternalistic leadership on voice behaviour - The moderating effect of mediocre thinking", Collected Essays on Finance and Economics, vol. 235, no. 7, pp. 88-96.

National Science Board, (2018), "Science and Engineering Indicators 2018", Retrieved from: https://www.nsf.gov/statistics/2018/nsb20181/

Nisbett, R. E., Peng, K., \& Choi, I., (2001), "Culture and systems of thought: Holistic versus analytic cognition", Psychological Review, vol. 108, no. 2, p. 291.

Ortiz-Avram, D., Domnanovich, J., \& Kronenberg, C., (2018), "Exploring the integration of corporate social responsibility into the strategies of small-and medium-sized enterprises: A systematic literature review”, Journal of Cleaner Production, vol. 201, pp. 254-271.

Pan, W., \& Sun, L. Y., (2018), "A self-regulation model of Zhong Yong thinking and employee adaptive performance", Management and Organization Review, vol. 14, no. 1, pp. 135-159.

Parkes, J., Hyde, C., \& Deeks, J. J., (2001), "Teaching critical appraisal skills in health care settings", Cochrane Database of Systematic Review, vol. 3, pp. 1-38.

Petticrewm, M., \& Roberts, H., (2008), Why do we need systematic reviews? Systematic reviews in the social sciences: A practical guide, John Wiley \& Sons, Oxford.

Qing, T., \& Liu, C., (2014), "Proactive personality and employees voice behaviour: Mechanisms of leader-member exchange and the role of Zhong Yang mentality", Journal of Sichuan University, vol. 1, pp. 127-134.

Qu, Y., Wu, W., \& Tang, F., (2018), "Why do I conform to your ideas?: The role of coworkers' regulatory focus in explaining the influence of Zhong Yong on harmony voice", Chinese Management Studies, vol. 12, no. 2, pp. 346-368.

Shen, Y., Ma, C., \& Bai, X., (2019), "Linking abusive supervision with employee creativity: The roles of psychological contract breach and Zhong Yong thinking style", Acta Psychologica Sinica, vol. 51, no. 2, pp. 238-247.

Shu, F., Julien, C. A., \& Larivière, V., (2019) "Does the web of science accurately represent chinese scientific performance?", Journal of the Association for Information Science and Technology, vol. 70, no. 10, pp. 1138-1152.

Snyder, H., (2019), "Literature review as a research methodology: An overview and guidelines", Journal of Business Research, vol. 104, pp. 333-339.

Spencer-Rodgers, J., \& Peng, K., (2017), The psychological and cultural foundations of East Asian cognition: Contradiction, change, and holism, Oxford University Press, Oxford.

Sun, X., Yan, M., \& Chu, X., (2014), "Passive mood and work behaviour: The cross-level mediating effect of Zhong-Yong thinking style", Acta Psychologica Sinica, vol. 46, no. 11, pp. 1704-1718.

Tranfield, D., Denyer, D., \& Smart, P., (2003), "Towards a methodology for developing evidence-informed management knowledge by means of systematic review", British Journal of Management, vol. 14, no. 3, pp. 207-222.

Vallaster, C., Kraus, S., \& Lindahl, J. M. M., (2019), "Ethics and entrepreneurship: A bibliometric study and literature review", Journal of Business Research, vol. 99, pp. 226-237.

Vrontis, D., \& Christofi, M., (2019), "R\&D internationalization and innovation: A systematic review, integrative framework and future research directions", Journal of Business Research, vol. ahead-of-print, pp. 1-12.

Wang, Q., \& Ngai, E. W., (2020), "Event study methodology in business research: A bibliometric analysis", Industrial Management \& Data Systems, vol. 120, no. 10, pp. 1863-1900.

Wei, J., (2019), "The influence mechanism of Zhong-Yong thinking pattern on employees' innovation behaviour", Economic Management in China, vol. 5, no. 7.

Wu, C. H., \& Lin, Y. C., (2005), "Development of a Zhong-Yong thinking style scale", Indigenous Psychological Research in Chinese Societies, vol. 24, pp. 247-300. 
Yang, C. F., (2010), "Multiplicity of Zhong Yong studies", Indigenous Psychological Research in Chinese Societies, vol. 34, pp. 93-96.

Yang, C. F., \& Chiu, C. Y., (1997), "A preliminary study on the practical thinking of Zhong Yong", The 4th Chinese Psychology and Behaviour Interdisciplinary Symposium, vol. 5, pp. 29-31.

Yang, Y., Jia, L., \& Liu, D., (2017), "The effect of perceived deep-level dissimilarities on employees' voice behaviour", Economic Management in China, vol. 4, pp. 97-112.

Yao, X., Yang, Q., \& Dong, N., (2010), "Moderating effect of Zhong Yong on the relationship between creativity and innovation behaviour", Asian Journal of Social Psychology, vol. 13, no. 1, pp. 53-57.

Yao, Y., \& Fan, Y., (2014), "The impact of person-organization fit on innovative behaviour The moderating effects of Zhong Yong thinking and Chaxu climate", East China Economic Management, vol. 28, no. 11, pp. 123-127.

Ye, Y., Omar, R., \& Ning, B., (2020a), "Exploring the interactions of factory workers in China: A model development using the grounded theory approach", Sustainability, vol. 12, no. 17 , p. 6750.

Ye, Y., Omar, R., \& Ning, B., (2020b), "Intergenerational transmission of occupation: A qualitative inquiry into frontline factory workers in China", Sustainability, vol. 12, no. 20, p. 8486.

Yuan, L., (2013), Traditional Chinese thinking on HRM practices: Heritage and transformation in China, Springer, London.

Zhang, G., \& Gu, X., (2015), "Moderation thinking and employees' creativity", Science Research Management, vol. S1, pp. 251-257.

Zhang, J., \& Long, L., (2016), "The influence of servant leadership on employee interpersonal citizenship behaviour: The role of forgiveness climate and Zhong-Yong thinking style", Journal of Industrial Engineering and Engineering Management, vol. 1, pp. 43-51.

Zhou, H., Xia, G., \& Deng, S., (2017), "The effects of error management atmosphere on employees' innovative behaviour - An analysis based on mean thinking as moderating variable", Commercial Research, vol. 4, pp. 115-121.

Zhou, S. S., Li, P. P., \& Zhou, A. J., (2019), "The cultural roots of compositional capability in China: Balanced moderation”, Asia Pacific Journal of Management, pp. 1-21. 